\title{
Fine-tuning the accretion disk clock in Hercules X-1
}

\author{
M. Still ${ }^{1}$ and P. Boyd ${ }^{2}$ \\ NASA/Goddard Space Flight Center, Greenbelt, MD 20771 \\ Martin.Still@gsfc.nasa.gov, padi@milkyway.gsfc.nasa.gov
}

\begin{abstract}
$R X T E$ ASM count rates from the X-ray pulsar Her X-1 began falling consistently during the late months of 2003. The source is undergoing another state transition similar to the anomalous low state of 1999. This new event has triggered observations from both space- and ground-based observatories. In order to aid data interpretation and telescope scheduling, and to facilitate the phase-connection of cycles before and after the state transition, we have re-calculated the precession ephemeris using cycles over the last 3.5 years. We report that the source has displayed a different precession period since the last anomalous event. Additional archival data from $C G R O$ suggests that each low state is accompanied by a change in precession period and that the subsequent period is correlated with accretion flux. Consequently our analysis reveals long-term accretion disk behaviour which is predicted by theoretical models of radiation-driven warping.
\end{abstract}

Subject headings: accretion, accretion disks — instabilities — ephemerides — (stars:) binaries: close X-rays: binaries - X-rays: individual (Her X-1)

\section{Introduction}

Her X-1 is an eclipsing X-ray pulsar that displays variability on spin $(1.24 \mathrm{~s})$, orbital $(1.7 \mathrm{~d})$ and super-orbital (35d) timescales (Tananbaum et al. 1972). The companion star is of early-F or late-A type and fills its Roche lobe, resulting in accretion mainly by Roche lobe overflow through the inner Lagrangian $\left(L_{1}\right)$ point (Leahy \& Scott 1998).

The $35 \mathrm{~d}$ period is revealed in X-rays as an absorbing column of variable depth passing in front of the accretion source (Scott, Leahy \& Wilson 2000), In the UV and optical, an intrinsic flux variation is observed on the same period (Gerend \& Boynton 1976; Leahy \& Marshall 1999). The long-standing model is of a warped accretion disk, viewed close to edge-on $(i \simeq 90 \mathrm{deg})$ precessing in a retrograde direction around the neutron star, (Katz 1973; Roberts 1974). The disk atmosphere provides the column and casts an X-ray shadow over the inner face of the companion star, causing optical variability on $35 \mathrm{~d}$ timescales.

\footnotetext{
${ }^{1}$ Universities Space Research Association.

${ }^{2}$ University of Maryland Baltimore County.
}

In X-rays, the $35 \mathrm{~d}$ cycle is characterized by a rapid turn-on, a "main-on" state which varies in intensity greatly from cycle to cycle, and then decays back to an "off" state at $\sim 1 \%$ of the peak main-on flux. This is followed by a rise to a weaker "short-on" state and then another low (Tananbaum et al. 1972; Gorecki et al. 1982). Superimposed on this cycle are eclipses of the neutron star by the companion once per orbit and dips that occur almost on the beat period between orbit and precession (Giacconi et al. 1973), which are believed to be due to localized structure in the disk, generated by the impact of the ballistic gas stream from the $L_{1}$ point (Crosa \& Bonton 1980; Schandl 1996). The turn-on occurs at only two orbital phases, $\phi_{\text {orb }} \simeq 0.2$ or $\phi_{\text {orb }} \simeq 0.7$, apparently at random. Consequently it has often been claimed that precession is not strictly periodic (Ogelman 1987), and precession cycles generally have durations of either $20,20.5$ or 21 orbital cycles, leading some authors to suggest that the precession and orbital cycles are related physically (Scott \& Leahy 1999).

To date, it has not been possible to determine 


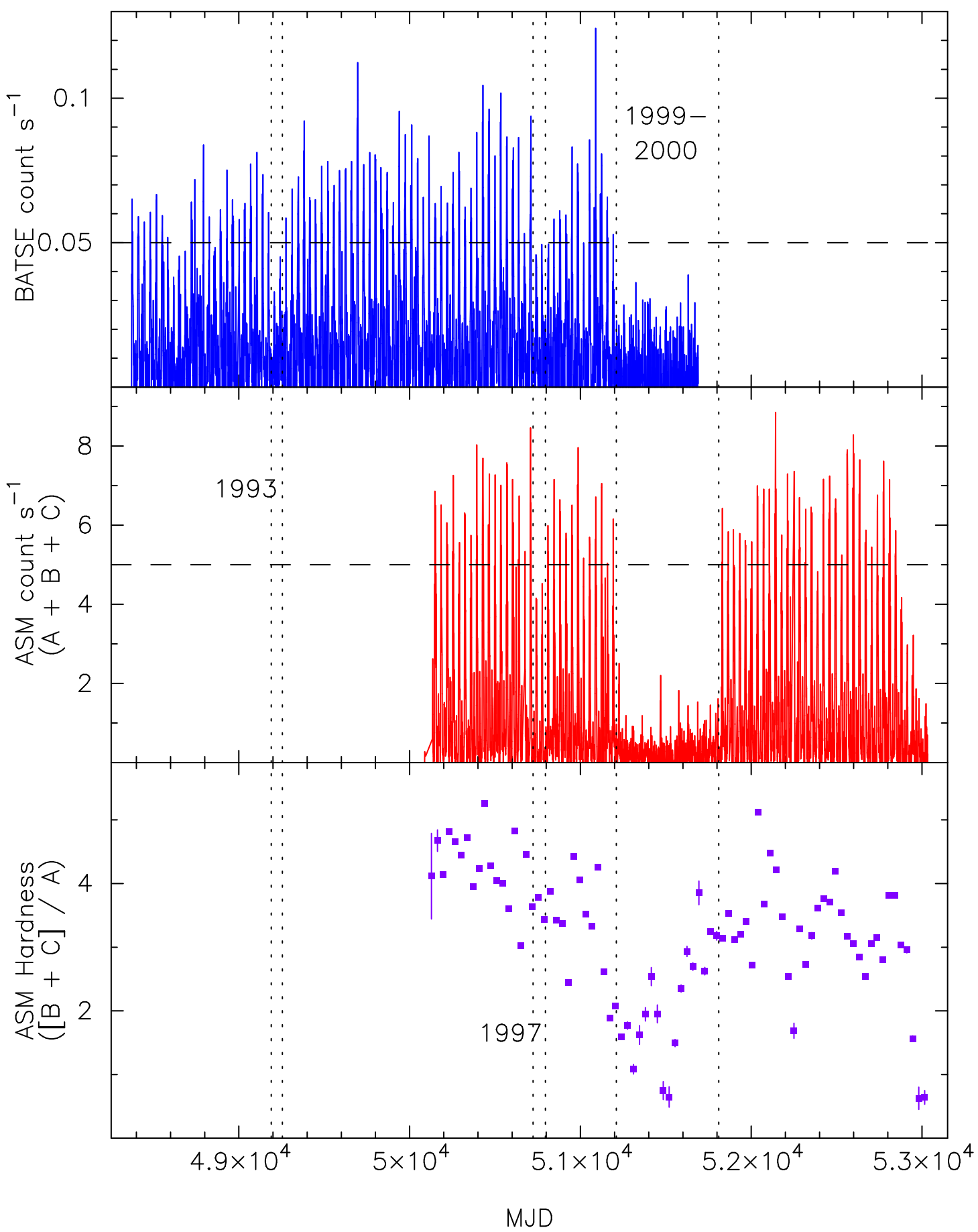

Fig. 1.- Top panel: Her X-1 CGRO BATSE (20-50 keV) one-day average count rates from Earth occultations between 1991-1999. Middle panel: RXTE ASM (1-12 keV) one-day average count rates from Her X-1 from 1996-2004. Bottom panel: ASM Hardness ratio averaged over each 35 day cycle. Band A $=1.3-3 \mathrm{keV}$, band $\mathrm{B}=3-5 \mathrm{keV}$ and band $\mathrm{C}=5-12 \mathrm{keV}$. Horizontal lines represent count rate thresholds adopted in the $0-C$ analysis. Dwells over the two thresholds are plotted on the $O-C$ diagram of Fig. 2 
the engine behind disk precession observationally. From a theoretical perspective, the companion star can force a tilted disk to precess in the outer regions closest to the donor star (Larwood 1998). However tidal forces do not account for the mechanism that tilts the disk. Mechanisms that can simultaneously warp structure and drive precession are wind (Schandl \& Meyer 1994) and radiation (Pringle 1996; Wijers \& Pringle 1999) pressure, pushing down on the disk plane. The dominant engine behind this pressure is accretion flux from the neutron star which irradiates the disk atmosphere externally. While the simplifying assumptions used in these theoretical calculations prevent a detailed comparison with observation, the success of the models in producing warped disks with the observed precession rate indicates that some combination of tides, wind and radiation pressure are capable of driving the Her X-1 clock.

Her X-1 has occasionally displayed consecutive main-on states of significantly lower flux than average, e.g. 1993 (Vrtilek et al. 1994). Also, between 1999-2000, the source missed a large number of consecutive cycles altogether (Parmar et al. 1999; Coburn et al. 2000; Vrtilek et al. 2001; Still et al. 2001). See also Parmar et al. (1985) for the description of a 1983 event prior to the epoch of all-sky monitoring surveys. During these times, the optical flux does not dip appreciably (Vrtilek et al. 2001). In addition to the decrease in main-on amplitude, the neutron star experiences an abrupt transition from the nominal state of pulse period spin-up to episodes of spin-down (Parmar et al. 1999). Although these events are not homogeneous in terms of either duration or magnitude, they have been coined collectively the 'Anomalous Low States (ALS)'. Quite clearly the ALS are significant disk events; they point to the accretion disk changing state for a limited period of time before returning seemingly to the same preferred period and warp shape, albeit with an offset of several orbital cycles in the turn-on clock, according to Oosterbroek et al. (2001) and Manchanda (2003). In this paper, we show this picture to be incorrect.

Rossi X-ray Timing Explorer (RXTE) All-Sky Monitor (ASM) count rates from Her X-1 in the main-on state began falling consistently during the late months of 2003. Her X-1 appears to be undergoing another state transition similar to the
ALS of 1999-2000. Since a glitch in the precession clock was reported after the last ALS (Oosterbroek et al. 2001), it is now prudent to recalculate the precession ephemeris using the last 3.5 years of data. Rather than a glitch, we report that the source has displayed a different precession period, $P_{35}$, since the last ALS. Additionally, archival data from the BATSE experiment that was onboard the Compton Gamma Ray Observatory (CGRO) suggests that each ALS is accompanied by a change in $P_{35}$. Contrary to previous reports (Oosterbroek et al. 2001), these results indicate that the accretion disk does not return to the same state after each ALS.

\section{Results}

Fig. 1 plots the total BATSE and ASM count rate as averages of all dwells taken within a $24 \mathrm{~h}$ period, weighted by uncertainty. Three ASM energy channels allow the source's spectral evolution to be monitored coarsely as a hardness ratio. The main-ons of the $35 \mathrm{~d}$ cycle reveal themselves as maxima in the count rate time series, where each cycle peaks at $3-9$ ASM count $\mathrm{s}^{-1}(1 \mathrm{Crab}=75$ count $\mathrm{s}^{-1}$ ). The stand-out features of this time series are the extended 1999 ALS between MJD 51 195-51892 (Parmar et al. 1999), and the decline into a putative new ALS starting MJD 52866 (Boyd \& Still 2004). The 1993 ALS reported by Vrtilek et al. (1994) can be seen as two consecutive main-ons with faint maxima of $<0.05$ BATSE count $\mathrm{s}^{-1}$ between MJD $49213-49282$.

The extended ALS also reveals itself in the hardness data. A soft spectrum in the ALS is consistent with that seen during normal low states of the 35d cycle (Scott \& Leahy 1999; Clarkson et al. 2003). There is some evidence that the hardness ratio returns to a normal level before the main-on cycle returns in count rate, and of long-term hardness evolution over the duration of ASM monitoring. For an analysis of the structure of ASM count rates, folded over the 35d cycle, see Shakura et al. (1998), Scott \& Leahy (1999) and Leahy (2002). A more detailed analysis of $35 \mathrm{~d}$ spectral evolution with GINGA is presented by Leahy (2001).

A period search over the ASM data obtained after the 1999 ALS between MJD 51 892-52866 using the Lomb-Scargle method (Scargle 1982) reveals a best period of $35.10 \pm 0.01 \mathrm{~d}$, with a false- 


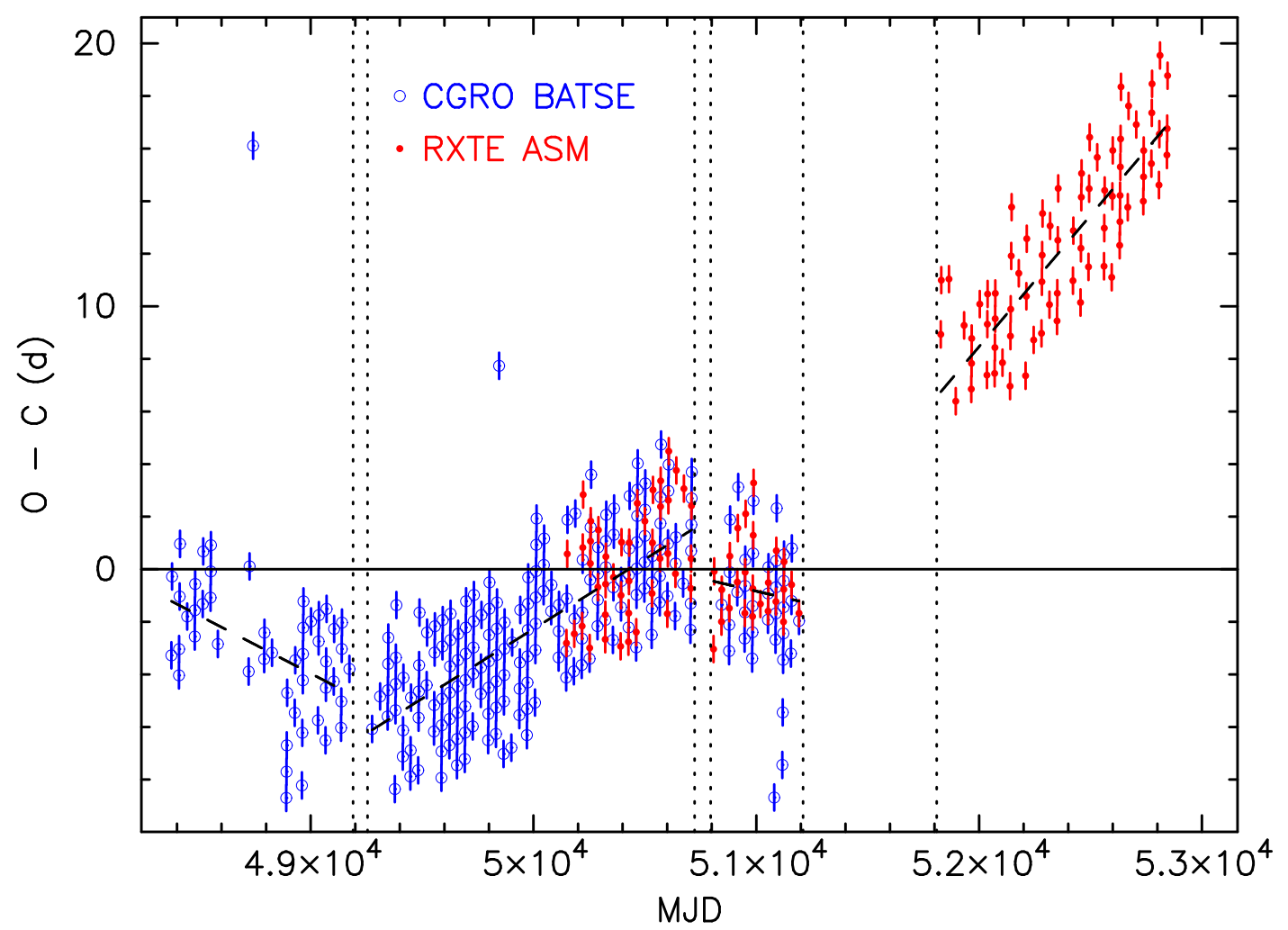

Fig. 2.- Observed - Computed time series of the main-on states in the Her X-1 precession cycle. Each point is a daily dwell in the time series that contains count rates above a threshold of 0.05 count s$^{-1}$ (BATSE) or 5 count $\mathrm{s}^{-1}$ (ASM). The reference ephemeris adopted is $T_{35}=50986.7+34.79 E_{35}$. Dashed lines represent linear least-square fits to the $O-C$ residuals between each ALS. Dotted vertical lines represent the start and stop times of each ALS.

Table 1: Precession periods, $P_{35}$, period derivative, $\dot{P}_{35}$, and maximum averaged main-on state count rates, $F_{\max }$, from four epochs of the Her X-1 precession cycle. Cycle number is relative to MJD 50 986.7, for consistency with Fig. 2.

\begin{tabular}{|c|c|c|c|c|c|c|c|}
\hline \multirow[t]{2}{*}{ Epoch } & \multicolumn{2}{|c|}{ Cycle } & \multicolumn{2}{|c|}{ MJD } & \multirow{2}{*}{$\begin{array}{l}P_{35} \\
\text { (d) }\end{array}$} & \multirow{2}{*}{$\begin{array}{c}P_{35} \\
\left(\mathrm{~d} \mathrm{~d}^{-1}\right)\end{array}$} & \multirow{2}{*}{$\begin{array}{c}F_{\max } \\
\left(\operatorname{count~s}^{-1}\right)\end{array}$} \\
\hline & Start & Stop & Start & Stop & & & \\
\hline 1 & -75 & -52 & 48377 & 49213 & $34.637 \pm 0.010$ & $-(3 \pm 1) \times 10^{-7}$ & $0.046 \pm 0.002^{\mathrm{a}}$ \\
\hline 2 & -49 & -8 & 49282 & 50708 & $34.977 \pm 0.003$ & $(3 \pm 2) \times 10^{-8}$ & $0.064 \pm 0.001^{\mathrm{a}}, 4.79 \pm 0.05^{\mathrm{b}}$ \\
\hline 3 & -5 & +6 & 50813 & 51195 & $34.745 \pm 0.021$ & $-(7 \pm 7) \times 10^{-10}$ & $0.042 \pm 0.003^{\mathrm{a}}$ \\
\hline 4 & +26 & +54 & 51892 & 52866 & $35.137 \pm 0.007$ & $(1 \pm 1) \times 10^{-7}$ & $5.00 \pm 0.06^{\mathrm{b}}$ \\
\hline
\end{tabular}

${ }^{a}$ CGRO BATSE

${ }^{b} R X T E$ ASM 
alarm probability $<10^{-6}$. This compares directly with an identical search over the pre-1999 ASM data, MJD $50813-51195$, of $34.79 \pm 0.03$ d. Clearly there is dichotomy here. To qualify this, we construct an "Observed - Computed" $(O-C)$ curve of precession cycles (Fig. 2). Since turn-on times are not strictly periodic, the simplest reference point for this analysis is the mid-point of each main-on state, $T_{\text {main. }}$. Any data point $>$ an arbitrary threshold of 0.05 (BATSE) and 5 (ASM) count $\mathrm{s}^{-1}$ is plotted on the $O-C$ diagram. So each individual main-on is represented by several data points in the diagram. Scatter is a relative measure of the duration of main-on states, although ASM epochs cannot be compared directly with BATSE epochs in this way because of the different flux thresholds. Our reference ephemeris, measured from all ASM data before the 1999 ALS, is $T_{\text {main }}=$ MJD $50986.7+34.79 E_{35}$, where $E_{35}$ is an integer. The quantity subtracted from BATSE and ASM times to produce the $O-C$ time series is the nearest $T_{\text {main }}$ to each daily dwell.

There are at least four epochs of different precession period evident in Fig 2. There is no evidence for episodes of high $\dot{P}_{35}$, except at discrete times that correspond to each ALS. The one exception is at MJD 50750 . There have been no reports of an ALS previously for this date, however we suggest that the low count rates and period change at this epoch qualify it as such. There is an unfortunate gap in the published Her X-1 pulse period history (Parmar et al. 1999) during this epoch where one would expect an episode of spin-down from the neutron star. Individual Lomb-Scargle searches and quadratic fits to the $O-C$ residuals provide independent, and consistent, measures of $P_{35}$ for each epoch. $O-C$ fits yield the smallest statistical error and we provide these in Table 1. Measured values of $\dot{P}_{35}$ are small during all epochs and these are also included in Table 1. Best fit ephemerides are plotted over the $O-C$ diagram as dashed lines, although we have made no attempt to align the ephemerides at the turning points. Based on our arbitrary count rate threshold, there are other candidates for ALS, particularly during the early BATSE era at MJD 48600. However, there is no evidence within the $O-C$ residuals or pulse history diagrams to confirm this.

Epoch-folding of the data over $P_{35}$, shows that turn-ons typically occur $0.4 \pi$ in phase before the mid-point of main-on, as defined by our threshold criteria, although there is an uncertainly of a few days because the turn-ons and maxima are decoupled. As discussed in Sec. 1, turn-ons appear not to be defined by precession period alone, also being dependent on orbital phase. Therefore, while we provide a precession ephemeris below for the turn-on times of epoch $4, T_{\text {on }}$, we must allow a systematic uncertainty of $\sim 1$ orbital cycle $(\sim$ $2 d)$ :

$$
T_{\text {on }}=\text { MJD } 51821(2)+35.10(1) E_{35}
$$

Fig. 3 plots the time series, separated by epoch as defined in Table 1, and folded over the relevant precession period. The data are averaged into 50 phase bins, where $\phi_{35}$ is the precession phase. $\phi_{35}=0$ is defined as the turn-on time. There are variations in the main-on structure from epoch to epoch, most noticeably in the peak flux of the main on state.

In Fig. 4 we plot main-on count rates, $F_{\max }$, versus precession period, $P_{35} . F_{\max }$ is measured as the peak of a Gaussian fit of the folded light curves in Fig. 3 between $\phi_{35}=0.0-0.4$. We find a positive correlation between $P_{35}$ and $F_{\max }$ in both the BATSE and ASM data, although the sample is, of course, very small, and the different energy bands of the two instruments means that they cannot be compared directly.

\section{Discussion}

Clarkson et al. (2003) noticed the period variation in the Her X-1 precession cycle at MJD 50750 , but interpreted it as a phase shift, suggesting that this event resulted eventually in the 1999 ALS, 400d later. From a longer baseline of data, we offer the alternative interpretation that each ALS is accompanied by a "simultaneous", change in $P_{35}$. The timescale of state changes must be just a few precession cycles. These are followed by epochs where $\dot{P}_{35} \simeq 0$, indicating long-lived, stable disk structure. Extrapolating the quadratic $O-C$ fit for epoch 4 , we find that an instantaneous $\dot{P}_{35}$ would have occurred towards the beginning of the 1999 ALS. A Gabor transform analysis (i.e. a short-time Fourier transform with a sliding window; Heil \& Walnut (1989), appropriate for uncovering evolution in frequency and amplitude, 


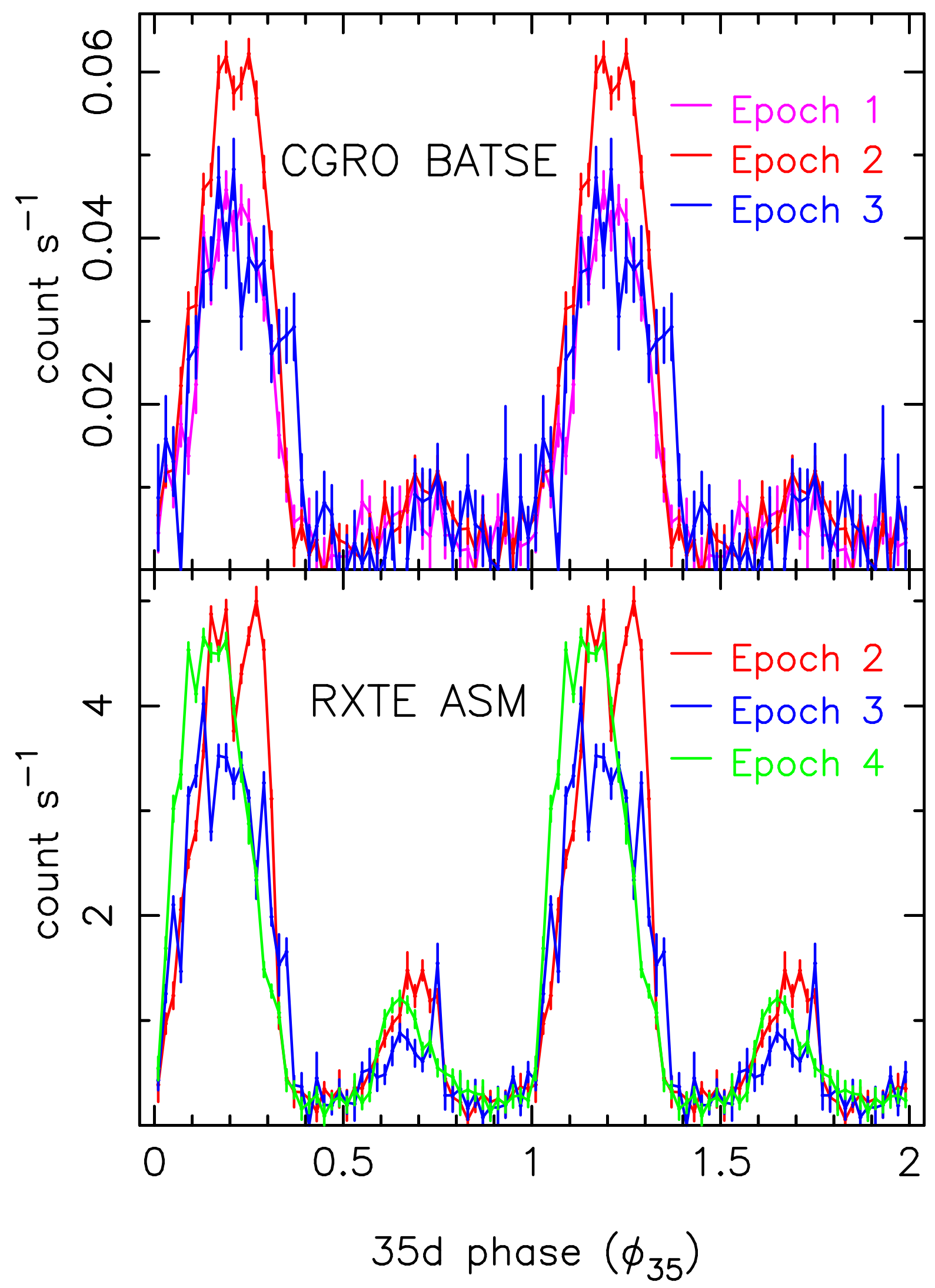

Fig. 3.- One-day BATSE and ASM dwells from each of the four epochs, folded over the epoch-dependent precession period and averaged into 50 phase bins. The epoch ranges are defined in Table 1 . The $35 \mathrm{~d}$ cycle is plotted twice for the interest of clarity. 


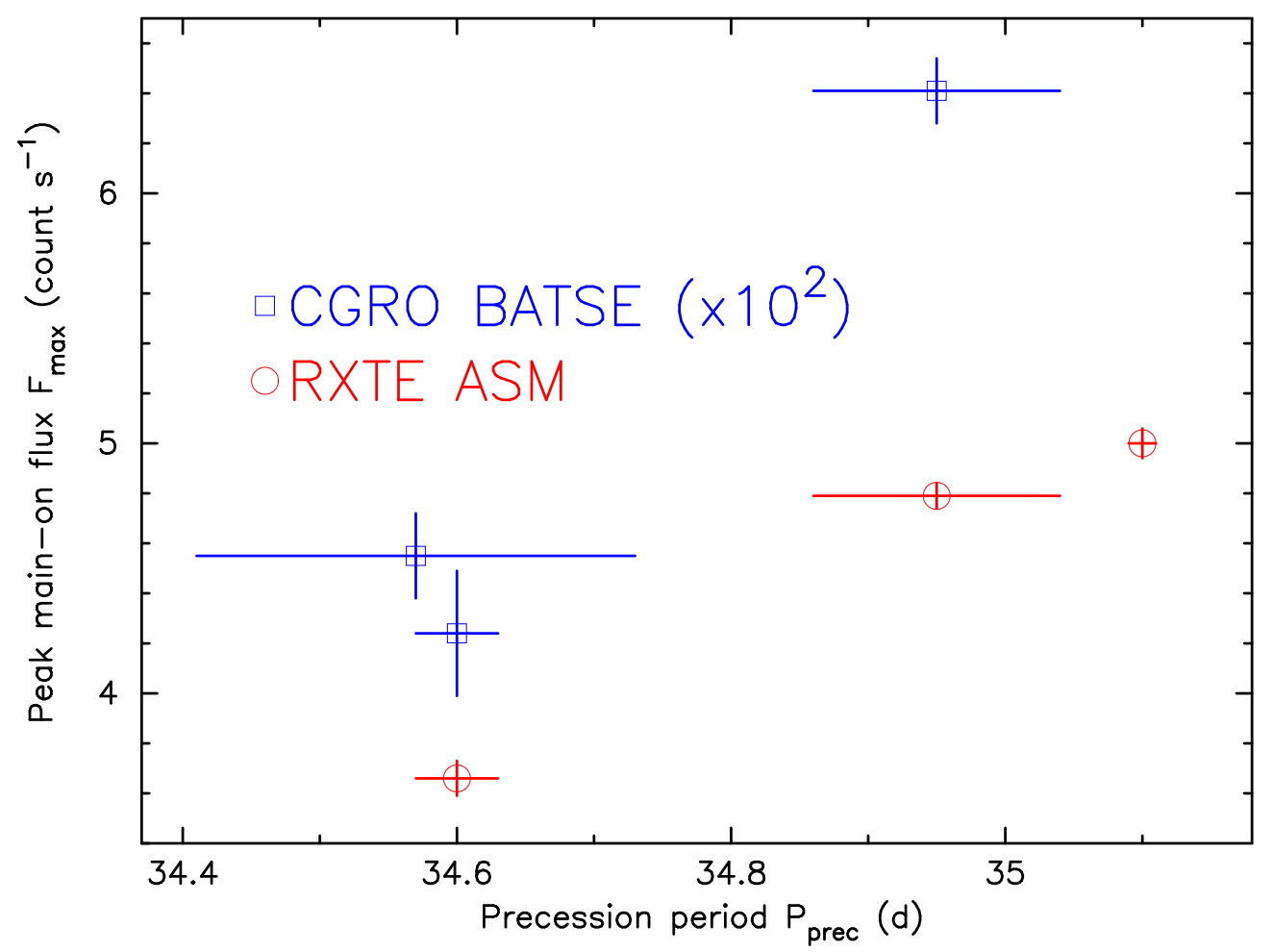

Fig. 4. - The time-averaged main-on BATSE and ASM count rates between $\phi_{35}=0.1-0.4$ as a function of precession period, $P_{35}$. Since the two instruments are sensitive to different energy ranges we have not renormalized one to the other, but have multiplied the BATSE count rates by $10^{2}$ to incorporate them on the same plot. No attempt is made to correlate BATSE fluxes with ASM fluxes due to the discrepent energy ranges and instrument characteristics between the two detectors.

resolves the ASM light curve of Her X-1 into a series of distinct episodes of constant period, consistent with the measurements reported in Table 1. However, due to the smaller sampling, the period uncertainties resulting from the Gabor transform analysis are much larger than those reported in Table 1. This explains why the three ASM precession epochs were not distinguishable in the dynamical power analysis of (Clarkson et al. 2003).

From Fig. 4, we can speculate that the mainon flux and precession period are related. At the peak of the main-on state, the absorbing column in front of the pulsar is consistent with the galactic column (dal Fiume et al. 1998), which does not contribute to the BATSE or ASM energy bands. It is reasonable, therefore, to assume that $F_{\max }$ measures the accretion flux from the pulsar (Scott, Leahy \& Wilson 2000). A relationship between $P_{35}$ and $F_{\max }$ is a natural prediction from models of radiation- and wind-driven precession (Schandl \& Meyer 1994; Wijers \& Pringle 1999), however an attempt to correlate the two quantitatively would require radiative and hydrodynamic models of enormous complexity. Wijers \& Pringle (1999) show that the precession timescale $\left(P_{\mathrm{d}}\right)$ is governed by:

$$
P_{\mathrm{d}} \sim \frac{\Sigma_{\mathrm{d}} R_{\mathrm{d}}^{3 / 2}}{L_{\mathrm{d}}}
$$

where $R_{\mathrm{d}}$ is a characteristic accretion disk radius, $\Sigma_{\mathrm{d}}$ a surface density and $L_{\mathrm{d}}$ is the strength of the radiation field from the disk. If the radiation field from the disk is dominated by irradiation from a central source then $F_{\max } \propto L_{\mathrm{d}}$. Since we find $F_{\max } \propto P_{35}$, Eqn. 2 is not consistent with observation. However disk warp is self-limiting; brighter than some threshold $L_{\mathrm{d} \text {,max }}$, the warp becomes so tightly wound that the disk is self-occulting and radiation pressure is reduced. In this phase space the precession period will be directly-correlated with the neutron star flux, because the surface area on the disk incident to the pulsar decreases with increasing warp. The simplest picture for Her X-1, therefore, is that the pulsar luminosity is close to the stability limit. This is consistent with the predictions from analytical modeling by Ogilvie \& Dubus (2001). 
A corollary from Eqn. 2 is that it is of no surprise that the precession period varies from epochto-epoch. In fact, it is predicted directly from theory (Wijers \& Pringle 1999; Ogilvie \& Dubus 2001). If structure extends to the outer disk edge, any alteration in disk tilt will result in the deposition of mass by the ballistic stream at a different range of accretion disk radii (Lubow 1989; Schandl 1996). The resulting redistribution of surface density over the disk will yield a new equilibrium configuration for the new epoch, according to Eqn. 2.

We have determined $P_{35}$ from the entire time series rather than the interval between consecutive main-on turn-ons. Since turn-ons occur at only orbital phases $\phi_{\text {orb }} \simeq 0.2$ and 0.7 , it is natural to assume that structure locked in the rotational frame of the binary is partly responsible for turn-on times (Schandl 1996). Measuring intervals between consecutive turn-ons would provide the same results as Sec. 2 only if a large number of cycles were sampled. As we have shown, $P_{35}$ has not remained stable for more than 40 consecutive cycles in the BATSE and ASM era. Consequently measuring turn-on intervals provides a more uncertain measure of $P_{35}$. One interesting direction for future work would be to determine whether turn-on times can be predicted from $P_{35}$, i.e., does the time of turn-on correspond to whichever orbital phase, 0.2 or 0.7 , immediately follows an undetermined reference phase within the stable precession cycle? Of course, the reference will change after each ALS.

\section{Conclusions}

We have redefined the accretion disk precession ephemeris for the X-ray pulsar Her X-1 between the end of the 1999-2000 ALS and the start of the 2003-2004 ALS event. Relative to the most recently published work on cycle timing (Manchanda 2003), the new ephemeris provides a correction to the turn-on time of the precession cycle of $\sim 14 \mathrm{~d}$ at the time of writing, critical to research teams and observatory schedulers during the current ALS. A period analysis of the CGRO BATSE and RXTE ASM time series since 1991 reveals four epochs of stable precession period. The precession epochs are each separated by an ALS and the change of period appears to be instantaneous within the resolution of the $O-C$ diagram, i.e. a few precession cycles. Testable predictions are that Her X-1 will return from the 2003-2004 ALS with a new precession period and epoch-averaged peak main-on flux.

Results provided by the $R X T E$ ASM teams at MIT and at the RXTE SOF and GOF at NASA's GSFC, and the CGRO BATSE instrument team at NASA's MSFC.

\section{REFERENCES}

Boyd, P., Still, M., 2004a, ATel, 228, 1

Clarkson, W. I., Charles, P. A., Coe, M. J., Laycock, S., 2003, MNRAS, 343, 1213

Coburn, W. et al., 2000, ApJ, 543, 351

Crosa, L., Boynton, P. E., 1980, ApJ, 235, 999

dal Fiume, D. et al., 1998, A\&A, 329, L41

Gerend, D., Boynton, P. E., 1976, ApJ, 209, 562

Giacconi, R., Gursky, H., Kellogg, E., Levinson, R., Schreier, E., Tananbaum, H. 1973, ApJ, 184,227

Gorecki, A. et al., 1982, ApJ, 256, 234

Heil, C. E., Walnut, D. F., 1989, SIAM Review, $31(4), 628$

Katz, J. I., 1973, Nature Phys. Sci, 246, 87

Larwood, J., 1998, MNRAS, 299, L32

Leahy, D. A., 2001, ApJ, 547, 449

Leahy, D. A., 2002, MNRAS, 334, 847

Leahy, D. A., Marshall, H., 1999, ApJ, 521, 328

Leahy, D. A., Scott, D. M. 1998, ApJ, 503 L63

Lubow, S. H., 1989, ApJ, 340, L1064

Manchanda, R. K., 2003, A\&A, 401, 1111

Ogelman, H., 1987, A\&A, 172, 79

Ogilvie, G. I., Dubus, G., 2001, MNRAS, 320, 485

Oosterbroek, T., Parmar, A. N., Orlandini, M., Segreto, A., Santangelo, A., Del Sordo, S., 2001, A\&A, 375, 922 
Parmar, A. N., Pietsch, W., McKechnie, S., White, N. E., Truemper, J., Voges, W., Barr, P., 1985, Nature, 313, 119

Parmar, A. N., Oosterbroek, T., Dal Fiume, D., Orlandini, M., Santangelo, A., Segreto, A., Del Sordo, S., 1999, A\&A, 350, L5

Pringle, J. E., 1996, MNRAS, 281, 357

Roberts, W. J., 1974, ApJ, 187, 575

Scargle, J. D., 1982, ApJ, 263, 835

Schandl, S., 1996, A\&A, 307, 95

Schandl, S., Meyer, F., 1994, A\&A, 289, 149

Scott, D. M., Leahy, D. A., 1999, ApJ, 510, 974

Scott, D. M., Leahy, D. A., Wilson, R. B., 2000, ApJ, 539, 392

Shakura, N. I.; Ketsaris, N. A.; Prokhorov, M. E.; Postnov, K. A., 1998, MNRAS, 300, 992

Still, M., O'Brien, K., Horne, K., Boroson, B., Titarchuk, L. G., Engle, K., Vrtilek, S. D., Quaintrell, H., Fiedler, H., 2001, ApJ, 554, 352

Tananbaum, H., Gursky, H., Kellogg, E. M., Levinson, R., Schreier, E., Giacconi, R. 1972, ApJ, 174, L143

Vrtilek, S. D. et al., 1994, ApJ, 436, L9

Vrtilek, S. D., Quaintrell, H., Boroson, B., Still, M., Fiedler, H., O'Brien, K., McCray, R., 2001, ApJ, 549, 522

Wijers, R. A. M., Pringle, J. E., 1999, MNRAS, 308, 207

This 2-column preprint was prepared with the AAS LATEX macros v5.2. 